\title{
A Study On Deep Learning And Machine Learning Techniques On Detection Of Parkinson's Disease
}

\author{
$P$. Mounika ${ }^{1, *}, S$. Govinda $\mathrm{Rao}^{2}$ \\ ${ }^{1}$ MTech Student, Computer Science and Engineering, GRIET, Hyderabad, Telangana, India. \\ ${ }^{2}$ Professor, Computer Science and Engineering, GRIET, Hyderabad, Telangana, India.
}

\begin{abstract}
Parkinson's disease (PD) is a sophisticated anxiety malady that impairs movement. Symptoms emerge gradually, initiating with a slight tremor in only one hand occasionally. Tremors are prevalent, although the condition is sometimes associated with stiffness or slowed mobility. In the early degrees of PD, your face can also additionally display very little expression. Your fingers won't swing while you walk. Your speech can also additionally grow to be gentle or slurred. PD signs and symptoms get worse as your circumstance progresses over time. The goal of this study is to test the efficiency of deep learning and machine learning approaches in order to identify the most accurate strategy for sensing Parkinson's disease at an early stage. In order to measure the average performance most accurately, we compared deep learning and machine learning methods.
\end{abstract}

\section{Introduction}

Parkinson's disease (PD) was first described by Dr. Parkinson's as "trembling paralysis". Parkinson's disease may be a brain disease that can cause tremor, firmness, and staggering, balance, and coordination. The symptoms of Parkinson's disease usually develop gradually and get degraded over time. As the disease progresses, people may find it difficult to walk and talk. You will also experience psychological and behavioral changes, as well as sleep problems, despondency, unmind fullness and fatigue. Parkinson's disease can affect both men and women. They are $50 \%$ more likely to suffer from this disease than women. The main risk factor for Parkinson's disease is aging. Although most people get Parkinson's disease after the age of 60 , there are still $5-10 \%$ of people who get Parkinson's disease before the age of 60.50 Called "early onset" disease.

PD occurs when nerve fibers or neurons near the brain that control gesture are damaged or die. These neurons usually release dopamine, which is an important neurotransmitter in the brain. When neurons die or become damaged, less dopamine is produced, which leads to exercise. Scientists are still not sure what causes the death of dopamine-producing cells. Actually, the amount of individuals suffering from PD has exceeded 10 million worldwide. Parkinson's disease starts with different kinds of tremors which are possibly hand tremors, limb rigidity and gait inconveniences. These tremors are generally distinguished into motor (i.e., movement related) and non-motor (i.e., non-movement related). Patients with motor symptoms are generally less affected than those with non-motor symptoms. Nonmotor symptoms include despondency, insomnia,
Anosmia, and cognitive ailment. It is important to remember that early spotting of Parkinson's disease can help control and improve symptoms. The early stages are critical to slow down progress and allow patients to receive disease-modifying drugs when they are available. Early detection of Parkinson' unwellness is important for speed the disease' course. Several data-driven methods are developed over the years to boost the identification of Parkinson' disease. Machine learning, with its data-driven methodologies, had brought an amendment within the templet within the manner essential info in metallic element biomarkers is retrieved and analysed, since it's latterly emerged as a possible topic of analysis in each academe and trade for the identification of PD. Furthermore, machine learning approaches give critical information that aids in the classification and diagnosis of Parkinson' disease, permitting quicker decision-making. so as to resolve the PD detection challenge, several machine learning algorithms are utilized in the literature. Dysphonia measures, for example, have been used to discriminate patients with Parkinson's disease from ablebodied ones. Because of its capability to extract nonlinearity by using nonlinear kernels, the support vector machine (SVM) is applied to solely four dysphonic options for palladium classification. On the idea of acoustic analysis of speech, 2 machine learning algorithms, Random Forest (RF) or Support Vector Machine (SVM), and a Convolutional neural network $(\mathrm{CNN})$, a deep learning model, are wont to determine Parkinson' disease. Random forest (RF), Support Vector Machine (SVM), and Convolutional Neural Network (CNN) results are compared. Deep learning algorithms have attracted specific attention in palladium diagnosing and getting high accuracy with no assumptions on

* Corresponding author: monika.pilla@gmail.com 
information distribution due to their ability to manage giant amounts of data.

Meticulous monitoring of the pre-motor stage in PD ensures early identification of PD. Rapid Eye Movement (REM) sleep Behavior Disorder (RBD) and sensory system loss are common symptoms of this pre-motor stage, which differ from typical motor symptoms. This paper ambitions to offer a comparative evaluation evaluating the maximum superior facts-pushed prediction tactics in figuring out PD inside the facts-pushed methodologies. Machine studying-primarily based totally and deep studying-primarily based totally facts-pushed methodologies are contrasted on this paper. A deep studying version is created to differentiate regular humans from humans with Parkinson's disease. The number one purpose of this studies is to offer a comparative evaluation and shed mild at the efficacy of superior prediction algorithms whilst implemented to small PD facts sets. Parkinson's Progression Markers Initiative provided the PD data used in this study (PPMI). The results showed that when compared to machine learning models, developed deep learning has a better detection performance in distinguishing normal persons from Parkinson's disease patients.

\section{Previous Work/Related Work}

There are many researches going on Parkinson's disease regarding the diagnosis at early stages and also symptoms identification through various techniques which can be useful for the initiation of therapeutic inventions at an early stage and also the strategies to manage them. Parkinson's disease can be analysed by choosing people from various platforms such as social media platforms, tele-monitoring of the disease using the vocal features and also by gathering clinical visits of elder people who frequently visit for check-ups with high charges. The major goal to be about distinguishing abled-bodies from those who have Parkinson's disease. Different categorization approaches are also compared for effective PD diagnosis.

[1], The Probabilistic Neural Network (PNN) is used in a study to distinguish persons with Parkinson's Disease from healthy ones. They primarily used three types of PNN models: incremental search (IS), Monte Carlo search (MCS), and hybrid search (hybrid search) (HS) and these models provided accuracies from $79 \%$ to $81 \%$ for diagnosis of the disease for the people who are not diagnosed before.

The data set they considered is comprised of Biomedical vocal measures which are gathered from 31 candidates among them 23 with the Parkinson's malady. The primary goal of information process is to tell apart folks with Parkinson' un-wellness from healthy people, with the "status attribute" being changed to non-PD for healthy people and atomic number 46 for candidates who are diagnosed with Parkinson' disease, leading to a classification downside with 2 decisions.

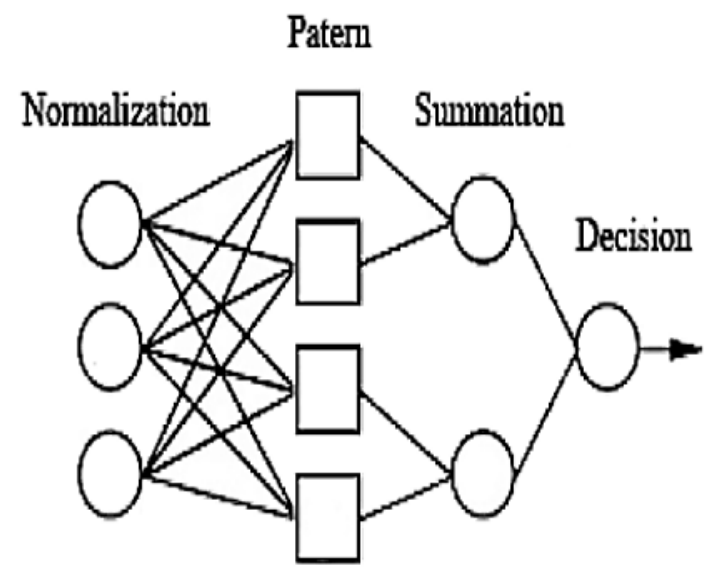

Figure 1. Neural Network Model (Ene, Marius 2008).

[2], The work presented here is an assessment of the value that is applied in practise to current traditional and non-traditional metrics for distinguishing people with PD from those who are healthy by detecting dysphonia. They also introduced Pitch Period Entropy (PPE), a new estimate for dysphonia which is used for detecting the variations in the frequency of voice. In their research they collected Phonations from few people among them most of the candidates with PD. The kernel support vector machine achieved a 91.4 percent performance. They finally concluded that noise ratios when combined with non-standard methods are the best methods to differentiate PD patients from healthy ones. Though the chosen non-standard methods are sturdy when subjected to variations in acoustic world they are well suited to telemonitoring applications.

[3], As in the previous work vocal features in measuring dysphonia play a major role and those features which are drawn out from voice are of various types and most of them co-related their research is two folded where 1.Selecting minimal subset of features and 2.Developing a low-bias predictive model. They used a interactive information metric with a permutation test to determine the pertinences. Also, in [2,] the standard deviation of a classifier's accuracy is exceptionally high $(35.84 \%)$, implying that testing on relatively small samples, such as 6 or 7 samples, yielded results of nearly $100 \%$ accuracy or virtually zero, indicating an inferior standard deviation. This is the signal indicating acquisition of the training set. And they finally concluded that their method generalizes better to unseen tests. As per the results, their method performs better even though the performance of SVM is inflated when compare to the study [2].And the set of all four high ranked features which are identified by [2] are poor and also vocal features possess higher capability in PD assessment.

[4], They in comparison many forms of categorization structures for correct prognosis of Parkinson's sickness on this study. Misdiagnosis is suggested to be excessive as $25 \%$ of the instances because the prognosis of PD could be very difficult.The purpose of their research is to describe the various approaches to efficiently differentiate healthy people. They used SAS base software for applying classification methods in the diagnosis of PD.A comparative study was carried out by 
applying 4 individual classification methods which are DMneural, Decision tree, Regression and Neural Networks. To compute the Performance of classification methods different evaluation methods are used. Their results proved that Neural Network classification model produces best outcomes and its overall performance is 92.9\%. They also compared their results to the outcomes that are yielded by kernel SVM's. This records highest classification score than the previous researches.

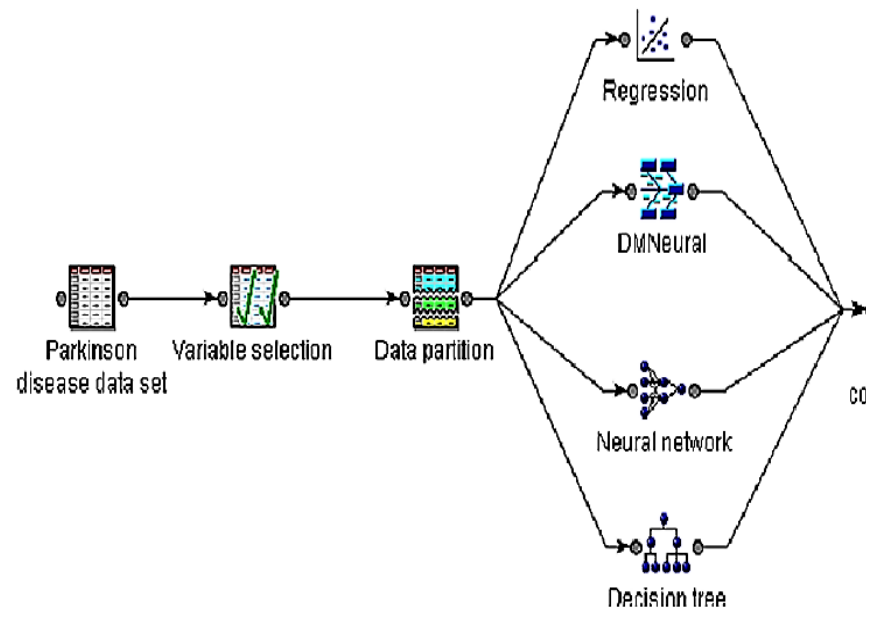

Figure 2. The applied methods for PD recognition (Das, Resul 2010).

[5], This work purpose is to differentiate the PD patients and the healthy individuals. Biomedical voice of human is used as Parkinson's dataset. Artificial Neural Networks (ANN) are wide utilized within the medical specialty trade for modelling, information analysis, and diagnostic classification. the 2 varieties of ANNs utilised for classification are Multilayer Perceptrons (MLP) and Radial Basis operate (RBF) Networks. The choice methodology for feature choice from the dataset is that the adaptational Neuro-Fuzzy Classifier (ANFC) with linguistic hedges. The simplest results from the adaptational Neuro-Fuzzy Classifier with linguistic hedges are $95.38 \%$ coaching and 94.72 percent testing.

[6], The aim of this observe is to apply fuzzy c-means (FCM) clustering-primarily based totally characteristic weighting to come across Parkinson's sickness (PD) (FCMFW). Practical values of current conventional and non-well known measures had been implemented to the enter of FCMFW to differentiate healthful humans from people with PD through figuring out dysphonia. The essential dreams of the FCM clustering set of rules are to convert a linearly non-separable dataset right into a linearly separable dataset and to enhance elegance discriminating performance. The weighted PD dataset comes with a k-nearest neighbour (k-NN) category model. The pleasant $\mathrm{k}$-cost became observed through searching at how k-values in a k-NN classifier modified while it became used to categorize Parkinson sickness datasets. Finally, they found that combining the proposed weighting approach dubbed FCMFW with the k-NN classifier produced the best results in the PD classification experiment.

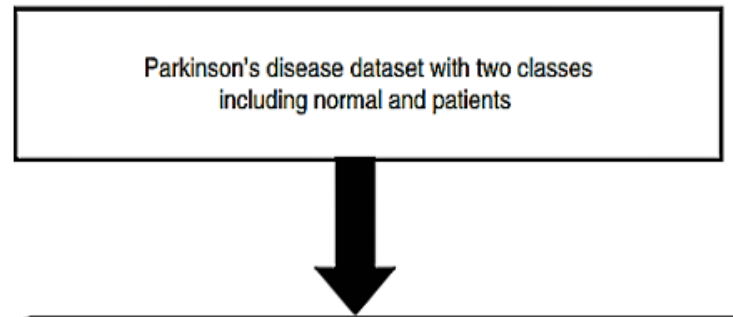

Weighting process the Parkinson's disease dataset using FCMFW Method

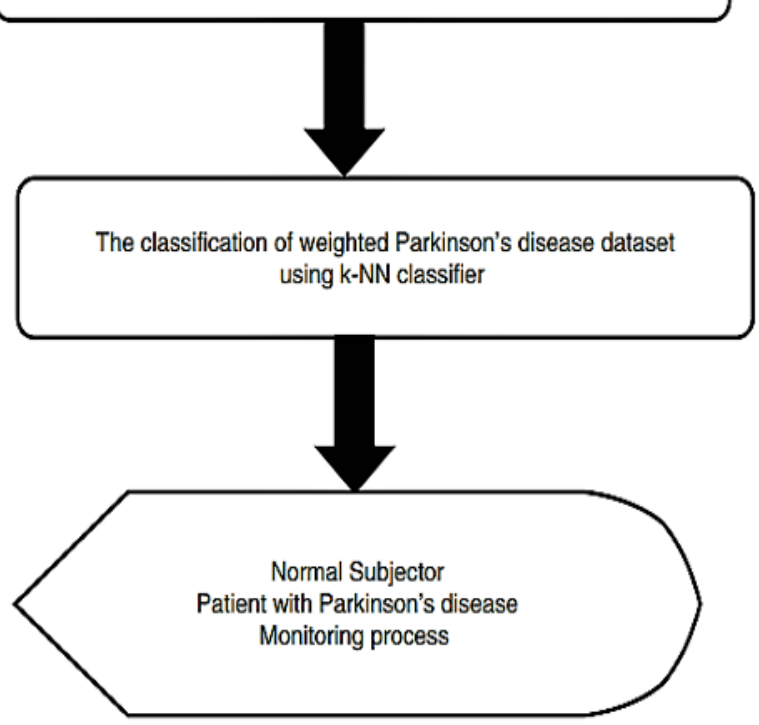

Figure 3. The block diagram of proposed method (Polat, Kemal 2012).

[7], In this study, feature selection is critical in classification to simplify the model and minimise the computational cost, especially when only a few inputs are required when the model is put to practical use. It's also utilised to make the model more transparent and understandable, allowing for a clearer explanation of potential diagnoses by removing irrelevant elements from the data set, which is a crucial necessity in medical applications. Noise can be decreased using the feature selection procedure to improve classification accuracy. They proposed a function choice method primarily based totally on fuzzy entropy measurements on this study, which turned into examined along a similarity classifier. The version is examined with 4 scientific statistics sets: dermatology, Pima-Indian diabetes, breast cancer, and Parkinson's disease. The researchers for this work tried to manage to get best results by only fewer features from the actual four data sets. Classification accuracy has been improved significantly with Parkinson's and dermatology data sets. With only two features out of 22 original ones, the imply type accuracy with Parkinson's statistics set is 85.03 percentage, and with dermatology statistics set, the imply accuracy turned into 98.28 percentage via way of means of using 29 capabilities in place of 34 authentic capabilities. Experiments have proven that once a function choice approach primarily based totally on fuzzy entropy measures is used with a similarity classifier, the outcomes are satisfactory.

[8], The study's important purpose is to apply a synthetic immune gadget to differentiate among 
wholesome folks and sufferers with Parkinson's disorder (PD). Tele-tracking of the disorder making use of dysphonia (vocal characteristics) checks is essential with inside the premature identity of Parkinson's disorder (PD), because the signs of the disorder rise up in aged folks for whom direct visits to the health facility are bulky and costly. They attempted to capture the valuable features of automatic identification, learning, and adaptation by drawing inspiration from natural immune systems. Training bio inspired CLONCLAS is the base algorithm that the developed algorithms have. Satisfiable results are obtained from the results showing the substantial reliability of approach. Several characteristics from the artificial immune systems are extracted from the experiments established in this work. Hybridization of the immune system algorithms with other approaches such as Neural Network or genetic algorithm is the future scope for this research.

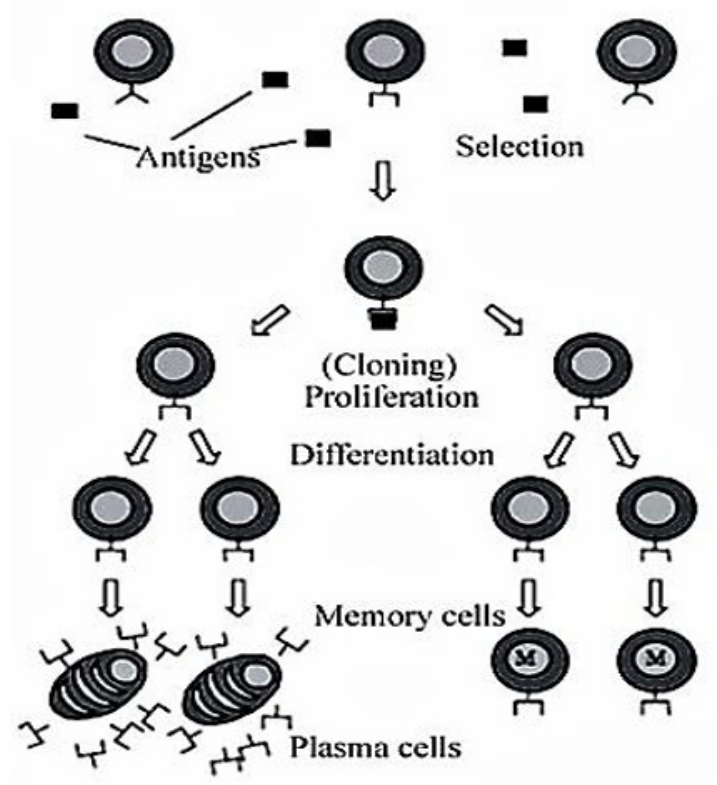

Figure 4. The clonal selection algorithm (Kihel, Badra Khellat, and Mohamed Benyettou 2011).

(8)

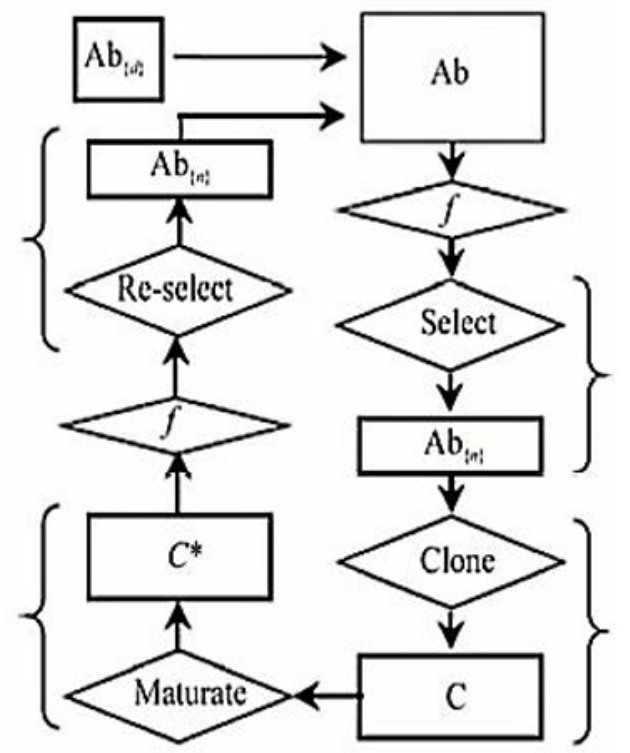

Figure 5. Clonclas train (Kihel, Badra Khellat, and Mohamed Benyettou 2011).
[9], Parkinson's disease is diagnosed using biomedical vocal measures acquired from incessant phonation samples. The six specific varieties of function choice techniques which are as compared of their studies to get the preferred effects are minimum-redundancy, maximum-relevancy(MRMR), Bhattacharyya, records gain, relief, t-test, and SVM (aid vector machine)techniques primarily based totally on recursive function elimination (SVM-RFE).Experiments conducted shown that $95.13 \%$ classification accuracy is obtained from SVM-RFE for Parkinson's disease dataset

[10], The degree and austerity of Parkinson's disease (PD) are essential standards to don't forget whilst making remedy selections. The mobility disorder Society-Unified Parkinson's Disease Rating Scale (MDS-UPDRS) is a beneficial device for assessing the maximum essential components of PD, but it does now no longer permit for PD staging. The Hoehn and Yahr (HY) scale offers staging, even though it does now no longer estimate numerous essential components of Parkinson's disease. They supplied a story and stronger staging for PD the usage of MDS-UPDRS characteristics, the HY scale, and developing prediction fashions on this study. They assessed the degree (normal, early, or moderate) and severity of PD the usage of ordinal logistic regression (OLR), guide vector machine (SVM), AdaBoost, and RUSBoost-primarily based totally classifiers. Aside from that, the price of Random forests functions in $\mathrm{PD}$ is estimated. The predictive fashions of SVM, Adaboost primarily based totally ensemble, Random forests, and probabilistic generative version that accomplished properly with the AdaBoost-primarily based totally ensemble had the nice accuracy of 97.forty six percentage of their studies. Body bradykinesia, tremor, facial [removed] hypomimia), fidelity of relaxation tremor, and handwriting are the maximum not unusualplace signs and symptoms of Parkinson's disease (micrographia). Finally, they located that once MDS-UPDRS is paired with classifiers, powerful equipment for predicting PD staging may be produced, which could resource therapists with inside the diagnostic process.

[11], Early identification of Parkinson's malady (PD) is regarded critical since it allows for the early implementation of treatment therapies and management methods. During their study period, however, approaches for early diagnosis remained an unmet clinical need in PD. The Patient Questionnaire (PQ) phase of the Movement Disorder Society-Unified Parkinson's Disease Rating Scale (MDS-UPDRS) is used for his or her study. As a result, gadget getting to know procedures together with logistic regression, random forests, boosted trees, and assist vector gadget (SVM) have become more and more more distinguished in biomedicine for classifying PD from healthful people at an early stage.

The researchers validate the machine learning approaches subject-by-subject and record-by-record. Their experiments classified early PD and healthy people with good accuracy and a large area under the ROC curve (both $>95 \%$ ). The logistic version indicates statistically large in shape to the data, suggesting its effectiveness as a prediction version. They determined that with the aid of using the usage of system mastering strategies to 
hyperlink the objects of a questionnaire, prediction fashions have the capacity to useful resource clinicians with inside the prognosis process.

[12], The primary aim of this look at is to examine and evaluate sentiment evaluation of sufferers with Parkinson's ailment the use of deep getting to know and phrase embedding fashions. This is the primary look at to apply phrase embedding fashions and deep getting to know algorithms to assess social media for Parkinson's ailment. Word2Vec, GloVe, and FastText are phrase embedding fashions that may be used to complement tweets in phrases of semantics, context, and syntax. These are used on this investigation. Convolutional Neural Networks (CNNs), Long Short-Term Memory Networks (LSTMs), and Recurrent Neural Networks (RNNs) are utilized to put into effect the type approach. It is concluded that analyzing people's attitudes with the aid of using explaining the performance of using phrase embedding fashions and deep getting to know algorithms affords a useful addition to the remedy technique with a 93.sixty three percentage accuracy performance. This work's researchers additionally aimed to enhance a hybrid version that integrates textual and vocal facts recording facts if you want to useful resource with inside the early detection of Parkinson's ailment.

[13], Preliminary detection of Parkinson's disease (PD) is essential to slow the progression of symptoms and ensure therapy for PD patients. The pre-motor stage of PD is carefully observed, and Deep Learning techniques are introduced based on these pre-motor traits to identify if an individual gets impacted with PD or not at an early stage. Many signs used for preliminary detection of Parkinson's disease include rapid eye movement and loss of smell, dopaminergic imaging markers, and cerebrospinal fluid data. The researchers compared the recommended deep learning model with few machine learning and collaborative learning methods that use a tiny amount of data. Including 183 healthy people and 401 early-stage Parkinson's disease patients, the developed model showed the best detection efficiency, with standard accuracy rate of $96.45 \%$. The consistency of the characteristics with the method of detecting EF is also determined by the amplification method. The research conducted in this study shows that the developed deep learning model is superior to 12 machine learning models used to distinguish between abled-bodies and Parkinson's disease patients.

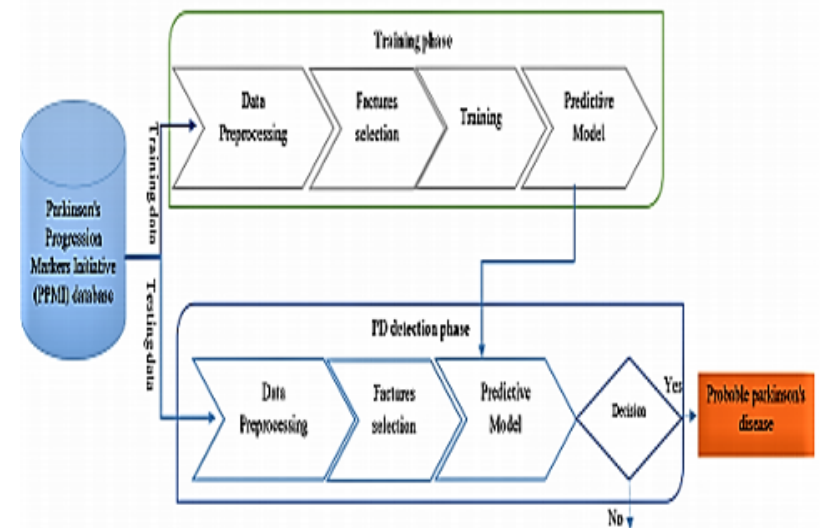

Figure 6. Flowchart of the proposed PD detection procedure (Wu Wang, Junho Lee, Fouzi Harrou \& Ying Sun).

\section{Comparison Study}

The Research works done on Detection of Parkinson's Disease are compared and differentiated in the below tabular format.

Table 1.Tabular Format to Compare and Differentiate the Research works done on PD.

\begin{tabular}{|c|c|c|c|}
\hline $\begin{array}{c}\text { Related } \\
\text { work }\end{array}$ & $\begin{array}{c}\text { Research } \\
\text { purpose }\end{array}$ & Algorithms & Accuracy \\
\hline$[1]$ & $\begin{array}{c}\text { PNN was } \\
\text { used to } \\
\text { distinguish } \\
\text { persons with } \\
\text { Parkinson's } \\
\text { Disease from } \\
\text { healthy } \\
\text { persons. }\end{array}$ & $\begin{array}{l}\text { Three different } \\
\text { PNN kinds } \\
\text { were } \\
\text { employed. } 3 \\
\text { hybrid search } \\
\text { 1. incremental } \\
\text { search (IS) } 2 \text {. } \\
\text { Monte Carlo } \\
\text { search (MCS) } \\
\text { 3. Hybrid } \\
\text { search (HS). }\end{array}$ & $\begin{array}{c}79 \%- \\
81 \%\end{array}$ \\
\hline [2] & $\begin{array}{c}\text { Pitch Period } \\
\text { Entropy (PPE) } \\
\text { is used to } \\
\text { distinguish } \\
\text { healthy } \\
\text { persons from } \\
\text { patients with } \\
\text { Parkinson's } \\
\text { disease (PD). }\end{array}$ & $\begin{array}{l}\text { Kernel support } \\
\text { vector machine } \\
\quad(\mathrm{SVM})\end{array}$ & $91.4 \%$ \\
\hline [3] & $\begin{array}{l}\text { (1) to choose } \\
\text { a limited } \\
\text { number of } \\
\text { features (2) to } \\
\text { develop a } \\
\text { bias-free } \\
\text { forecasting } \\
\text { model }\end{array}$ & $\begin{array}{c}\text { Machine to } \\
\text { Support } \\
\text { Vectors (SVM) }\end{array}$ & $92.75 \%$ \\
\hline [4] & $\begin{array}{l}\text { to efficiently } \\
\text { distinguish } \\
\text { healthy } \\
\text { individuals } \\
\text { from PD } \\
\text { patients }\end{array}$ & $\begin{array}{l}\text { DMneural, } \\
\text { Regression, } \\
\text { Decision Tree } \\
\text { and neural } \\
\text { networks. }\end{array}$ & $92.9 \%$ \\
\hline$[5]$ & to distinguish & There were two & $95.38 \%$ \\
\hline
\end{tabular}




\begin{tabular}{|c|c|c|c|}
\hline & $\begin{array}{c}\text { between } \\
\text { persons who } \\
\text { are healthy } \\
\text { and those who } \\
\text { have } \\
\text { Parkinson's } \\
\text { disease }\end{array}$ & $\begin{array}{l}\text { types of ANNs } \\
\text { employed in } \\
\text { this study. } \\
\text { 1.Multilayer } \\
\text { Perceptrons } \\
\text { (MLP) and } 2 . \\
\text { Radial Basis } \\
\text { Function } \\
\text { (RBF) } \\
\text { Networks }\end{array}$ & $\begin{array}{l}\text { training } \\
\text { and } \\
94.72 \% \\
\text { testing }\end{array}$ \\
\hline [6] & $\begin{array}{l}\text { To identify } \\
\text { between } \\
\text { persons who } \\
\text { are healthy } \\
\text { and those who } \\
\text { have } \\
\text { Parkinson's } \\
\text { disease }\end{array}$ & $\begin{array}{c}\text { FCMFW and } \\
\text { k-NN } \\
\text { classifiers }\end{array}$ & $\begin{array}{c}72.16 \% \\
\text { (raw PD } \\
\text { dataset), } \\
97.93 \\
\text { (weighted } \\
\text { PD } \\
\text { dataset) }\end{array}$ \\
\hline [7] & $\begin{array}{c}\text { Fuzzy entropy } \\
\text { measures are } \\
\text { used to pick } \\
\text { features. }\end{array}$ & $\begin{array}{l}\text { Classifier for } \\
\text { similarity }\end{array}$ & $98.28 \%$ \\
\hline [8] & $\begin{array}{l}\text { Utilizing the } \\
\text { artificial } \\
\text { immune } \\
\text { system to } \\
\text { distinguish } \\
\text { between } \\
\text { healthy ones } \\
\text { and } \\
\text { individuals } \\
\text { with } \\
\text { Parkinson's } \\
\text { disease (PWP) }\end{array}$ & $\begin{array}{l}\text { Bio inspired } \\
\text { CLONCLAS }\end{array}$ & $\begin{array}{l}87.50 \%, \\
88.54 \%\end{array}$ \\
\hline [9] & $\begin{array}{l}\text { To compare } \\
\text { feature } \\
\text { selection } \\
\text { strategies for } \\
\text { Parkinson's } \\
\text { malady } \\
\text { diagnosis on } \\
\text { vocal } \\
\text { measures. }\end{array}$ & $\begin{array}{l}\text { Bhattacharyya, } \\
\text { information } \\
\text { gain, relief, } \\
\text { minimum- } \\
\text { redundancy } \\
\text { maximum- } \\
\text { relevancy } \\
\text { (MRMR), t- } \\
\text { test, } \\
\text { SVM,SVM- } \\
\text { RFE }\end{array}$ & $95.13 \%$ \\
\hline$[10]$ & $\begin{array}{l}\text { Using the } \\
\text { MDS-UPDRS } \\
\text { features and } \\
\text { the HY scale, } \\
\text { present a new } \\
\text { and better PD } \\
\text { staging } \\
\text { system. }\end{array}$ & $\begin{array}{c}\text { Random } \\
\text { forests, SVM, } \\
\text { Adaboost based } \\
\text { ensemble, and } \\
\text { probabilistic } \\
\text { generative } \\
\text { models. }\end{array}$ & $97.46 \%$ \\
\hline [11] & $\begin{array}{l}\text { to create } \\
\text { prediction } \\
\text { models that } \\
\text { can } \\
\text { distinguish } \\
\text { between early } \\
\text { PD and } \\
\text { healthy } \\
\text { normal }\end{array}$ & $\begin{array}{c}\text { Random } \\
\text { forests, boosted } \\
\text { trees, and } \\
\text { support vector } \\
\text { machines } \\
(\mathrm{SVM}) \text { and } \\
\text { logistic } \\
\text { regression }\end{array}$ & $>95 \%$ \\
\hline$[12]$ & $\begin{array}{l}\text { Using deep } \\
\text { learning and } \\
\text { word } \\
\text { embedding } \\
\text { models, } \\
\text { analyse and }\end{array}$ & $\begin{array}{c}\text { Long Short- } \\
\text { Term Memory } \\
\text { Networks } \\
\text { (LSTMNs), } \\
\text { Convolutional } \\
\text { Neural }\end{array}$ & $93.63 \%$ \\
\hline
\end{tabular}

\begin{tabular}{|c|c|c|c|}
\hline & compare & Networks & \\
people's & (CNNs), and & \\
sentiment & Recurrent & \\
& analysis & Neural & \\
concerning & Networks & \\
& Parkinson's & (RNNs) and & \\
& disease. & Long short- & \\
& & term memory & \\
& & networks & \\
& & (LSTMs) & \\
\hline$[13]$ & to determine & twelve machine & \multirow{2}{*}{ le.45\% } \\
& if a person has & learning and & \\
& Parkinson's & ensemble & \\
& disease or not & learning & \\
& based on pre- & methods, as & \\
& motor & well as a deep & \\
& characteristics & learning model & \\
\hline
\end{tabular}

From the above comparison table,

In the study [1], PNN was used to distinguish persons with Parkinson's Disease from healthy persons. Three different PNN kinds were employed 1. incremental search (IS) 2. Monte Carlo search (MCS) 3. Hybrid search (HS). Among these Hybrid Search has the best accuracy with Training accuracy as $(81.74 \%)$ Testing accuracy as $(81.28 \%)$.

In the study [2], Pitch Period Entropy (PPE) is used to distinguish healthy persons from patients with Parkinson's disease (PD). $91.4 \%$ of classification performance is achieved through kernel SVM.

In the study [3], the research purpose is (1) to choose a limited number of features (2) to develop a bias-free forecasting model. $92.75 \%$ of accuracy is achieved through SVM.

In the study [4], the purpose of the research is to efficiently distinguish healthy individuals from PD patients by using classification methods like DMneural, Regression, and Decision Tree and neural networks. $92.9 \%$ of classification accuracy is achieved through neural networks from the above four classification methods.

In the study [5], There were two types of ANNs employed Networks 1. Multilayer Perceptrons (MLP) and 2. Radial Basis Function (RBF) Networks. 95.38\% training and $94.72 \%$ testing accuracies are achieved for the method ANFC-LH.and the total classification accuracy achieved through ANFC-LH is $96.77 \%$.

In the study [6], FCMFW and k-NN classifiers are used. FCMFW has achieved classification accuracies $72.16 \%$ with raw PD dataset and $97.93 \%$ with weighted PD dataset.

In the study [7], Fuzzy entropy measures are used to pick features. $98.28 \%$ mean accuracy is achieved through similarity classifier with 29 features.

In the study [8], by Utilizing the artificial immune system to distinguish between healthy ones and individuals with Parkinson's disease (PWP) the Bio inspired CLONCLAS is trained and the results shown are $100 \%, 100 \%$ as training accuracies and $87.50 \%$ and $88.54 \%$ as testing accuracies for Euclidian and hamming affinities respectively.

In the study [9], To compare feature selection strategies for Parkinson's malady diagnosis on vocal 
measures the methods used are Bhattacharyya, information gain, relief, minimum-redundancy maximum-relevancy (MRMR), t-test, SVM, SVM-RFE. $95.13 \%$ is the best accuracy achieved through SVM-RFE.

In the study [10], the purpose is Using the MDSUPDRS features and the HY scale and to present a new and better PD staging system. The methods used are Random forests, SVM, Adaboost based ensemble, and probabilistic generative model are all examples of probabilistic generative models. $97.46 \%$ is the highest accuracy achieved through Adaboost algorithm.

In the study [11], the purpose is to create prediction models that can distinguish between early PD and healthy normal. The methods used are Random forests, boosted trees, and support vector machines (SVM's) and logistic regression. All these methods produce highest accuracy and highest with both greater than $95 \%$ under the curve ROC.

In the study [12], the purpose is, using deep learning and word embedding models, analyse and compare people's sentiment analysis concerning Parkinson's disease. Long Short-Term Memory Networks (LSTMNs), Convolutional Neural Networks (CNNs), and Recurrent Neural Networks (RNNs) and Long short-term memory networks (LSTMs). 93.63\% of performance is achieved by utilizing word embedding models and deep learning models in sentiment analysis.

In the study [13], the purpose is to determine if a person has Parkinson's disease or not based on pre-motor characteristics. Twelve machine learning and ensemble learning methods, as well as a deep learning models are used. $96.45 \%$ is the highest average accuracy achieved on all the models.

In all the above research works the main focus is to distinguish parkinson's subjects from the healthy ones majorly. But the study [13] focuses mainly on early detection of PD and feature importance. Though study [13] focuses on early detection there is no particular method proved to give the best results. So, the future work can be focused on the research of the specific method which gives highest performance when different types of methods such as deep learning models, ensembling models and machine learning models are considered.

The following graph shows the accuracy for the research works contributed before for diagnosing PD using various approaches and various methods as described in the above tabular form in correspondence with the study.

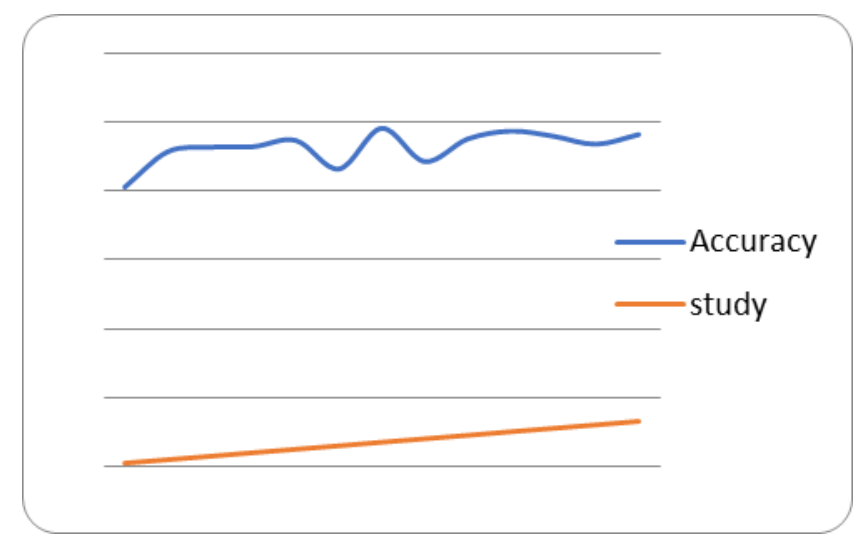

Figure 7. Graph showing accuracy in correspondence with studies from [1] to [13] in PD detection.

\section{Conclusion/Future Work}

According to the literature review, earlier research on Parkinson's Disease (PD) has primarily focused on distinguishing healthy persons from Parkinson's Disease sufferers. PNN, SVMs, Regression models, Decision trees, artificial immune systems (CONCLAS), ANN, k$\mathrm{NN}$, FCMFW, and other methods are utilised to distinguish healthy people from PD patients. And additionally models are developed to spot Parkinson's unwellness at AN early stage corresponding to SVM's, CNN, RNN, LSTM's, ensemble strategies and so on it's inferred that within the recent works published, although numerous methods are used for detective work palladium at an early stage, the performance measures may be valid as a key side in with efficiency distinctive the palladium at an early stage.So, the future work can be contributed with enhanced performance measures than the previous ones depicting that it was the best desired model in PD detection. Apart from this feature selection and feature importance can also be considered as the major scope of work to be concentrated as considerably less work is contributed in this aspect. The main focus of future work can be on evaluating the performance metrics for the Machine learning models, deep learning models and some ensembling techniques and the best performance is to be evaluated by considering the evaluation metrics and the method which gives highest accuracy can be considered as the best method in early detection of PD disease. Feature importance is also to be concentrated for future work as it has broader scope in research aspects in detecting PD accurately.

\section{References}

[1] Ene, Marius. Annals of the University of Craiova-Mathematics and Computer Science Series 35, 112 (2008).

[2] Little, M. A., McSharry, P. E., Hunter, E. J., Spielman, J., \& Ramig, L. O, IEEE transactions on bio-medical engineering, 56, 1015 (2009).

[3] Sakar, C. Okan, and Olcay Kursun. Journal of medical systems 34, 591 (2010). 
[4] Das, Resul. Expert Systems with Applications, 37, 1568 (2010).

[5] Caglar, Mehmet Fatih, Bayram Cetisli, and Inayet Burcu Toprak. Journal of Engineering Science and Design 1, 59 (2010).

[6] Polat, Kemal. International Journal of Systems Science 43, 597 (2012).

[7] Luukka, Pasi. Expert Systems with Applications, 38, 4600 (2011).

[8] Kihel, Badra Khellat, and Mohamed Benyettou. JSEA 4, 391 (2011).

[9] Eskidere, Ö. Sigma, 30, 402 (2012).

[10] Prashanth, R., \& Roy, S. D. Neurocomputing, 305, 78 (2018).

[11] Prashanth, R., \& Roy, S. D. International journal of medical informatics, 119, 75 (2018).

[12] Çevik F, Kilimci ZH. Academic Perspective Procedia. 2, 786 (2019).

[13] Wang W, Lee J, Harrou F, Sun Y. IEEE Access. 8, 147635 (2020). 